\title{
Evidence for a conceptual account of same-different discrimination learning in the pigeon
}

\author{
MICHAEL E. YOUNG \\ Southern Illinois University, Carbondale, Illinois \\ and \\ EDWARD A. WASSERMAN \\ University of Iowa, Iowa City, Iowa
}

\begin{abstract}
We trained pigeons to peck two different buttons in response to 16-icon same arrays versus 16-icon different arrays. In the same arrays, the icons were all the same as one another, whereas in the different arrays, the icons were all different from one another. In Experiment 1, we upset the spatial regularities of the displays by disarranging the icons-randomly displacing each icon to reduce the degree of perceptual order. The pigeons' discriminative performance was unaffected by disarranging. In Experiment 2, spatial regularities were disturbed by varying the rotation of the icons within a display. Again, no disruption in discriminative performance was observed. These and other findings suggest that pigeons treat the 16 icons as either the same or different despite changes in the spatial organization or orientation of the icons, thus implicating a conceptual rather than a perceptual process in same-different discrimination.
\end{abstract}

In 1995, Wasserman, Hugart, and Kirkpatrick-Steger reported that pigeons learned to discriminate 16 -item arrays of identical black-and-white computer icons from 16-item arrays of nonidentical computer icons (Figure 1). The pigeons not only discriminated same from different arrays that were created from the set of 16 training icons, but they also discriminated additional same and different arrays that were created from a set of 16 novel testing icons. These data suggest that the pigeons had acquired an abstract concept that depended on the relationship among the display items, and not on the particular items themselves.

But the same and the different arrays that are depicted in Figure 1 also differ in their spatial orderliness; the same arrays possess horizontal and vertical regularities that the different arrays lack. This could be considered a perceptual rather than a conceptual distinction.

In order to determine whether spatial orderliness is necessary for pigeons to discriminate same from different arrays, Young and Wasserman (1997, Experiment 1) trained pigeons to discriminate 16-item same arrays from 16-item

This research was supported by Research Grant IBN 99-04569 from the National Science Foundation. We thank Brian Nolan and Jessie Peissig for their assistance in the collection of the data. Correspondence concerning this article should be addressed to M. E. Young, Department of Psychology, Southern Illinois University, Carbondale, IL 62901 (e-mail: meyoung@siu.edu). different arrays, in which the items were randomly placed in a subset of the 25 locations of a $5 \times 5$ grid (see Figure 2). The pigeons readily acquired this discrimination, and they showed excellent transfer to additional arrays that were created from a novel set of icons.

Although placing the 16 icons into the 25 grid locations did disrupt some of the spatial orderliness of the displays, other regularities remained, particularly when adjacent items appeared in the rows and columns of the same arrays. Still, our prior work suggested that the pigeons may not use spatial orderliness as the basis of their same-different discrimination. Two pieces of evidence are relevant.

First, in a transfer test to arrays of novel icons (Young \& Wasserman, 1997), we found not only that our pigeons evidenced good discriminative transfer, but also that there was a statistically significant drop in accuracy from the familiar to the novel icon arrays from $93 \%$ to $79 \%$ (see also Wasserman et al., 1995). This generalization decrement suggests that the pigeons did attend to the individualicons in the arrays; if the pigeons had attended solely to spatial orderliness, changes in icon identity should have had no effect on performance. It is nevertheless possible that the pigeons may have attended to both icon identity and spatial orderliness.

Second, we conducted another study in which we showed pigeons lists of same and different icons on a one-at-a-time basis (a successive same-different discrimination; Young, 

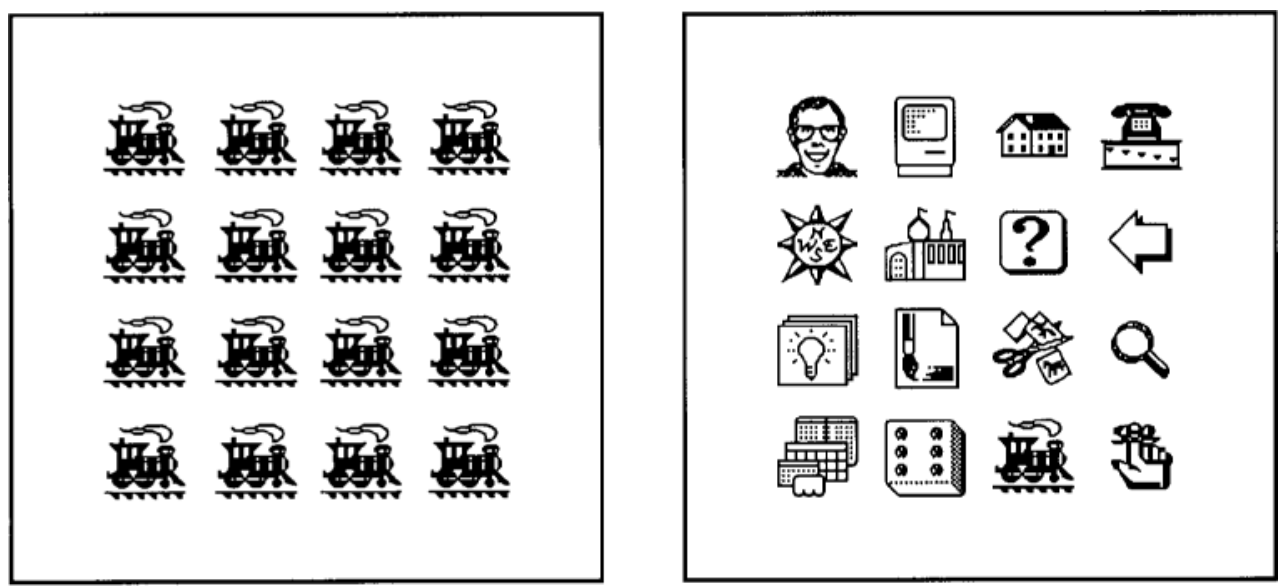

Figure 1. Trained same and different displays involving 16 icons distributed within a $4 \times 4$ array.

Wasserman, \& Dalrymple, 1997). Here, only one icon at a time was present on the screen, thereby making spatial orderliness an unusable cue for solving the same-different discrimination. The pigeons again exhibited excellent discriminative performance. Although we clearly demonstrated that pigeons can solve a successive same-different task in the absence of differences in spatial orderliness, this result cannot determine whether pigeons solve a $s i$ multaneous same-different discrimination by using such stimulus differences.

Rather than a perceptual account of pigeons' same-different discrimination that relies on spatial regularities, our conceptual account suggests that pigeons categorize each type of icon and determine its frequency in computing an array's entropy. Entropy is a measure of categorical variability: If there is a large variety of items, variability is high; if there is little variety, entropy is low. For example, if one's bird feeder attracts only sparrows and wrens, the variety of birds attracted is low, as reflected in a low entropy score for bird type; but if the bird feeder attracts a wide variety of bird species, entropy is higher.

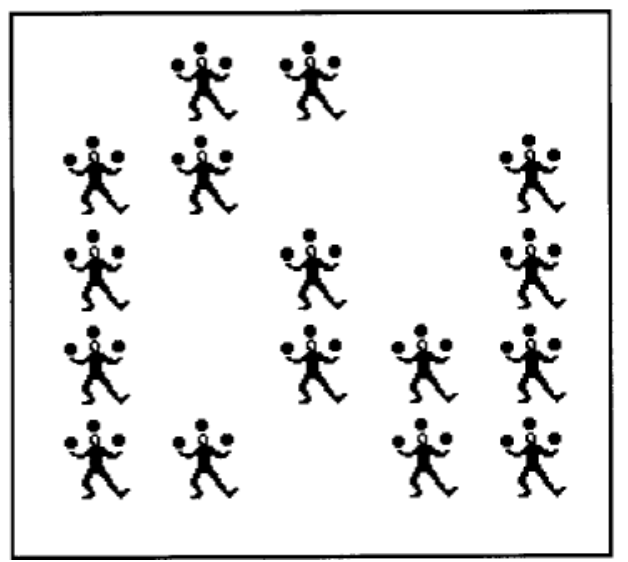

To determine categorical variability, it is necessary to assign each item to a category. In the bird feeder example, the category choice was at a level that distinguished sparrows from wrens from cardinals from finches, for example; but the level of categorization chosen affects the computation of entropy. The entropy score would be lower if the bird feeder visitors were categorized at a higher, more abstract level (e.g., squirrel and bird), and entropy would be higher if visitors were categorized at a lower, more detailed level (e.g., house sparrow, grasshopper sparrow, or Lincoln's sparrow).

The need for icon categorization is clear in the computational formula for entropy (Shannon \& Weaver, 1949):

$$
H(A)=-\sum_{a \in A} p_{a} \log _{2} p_{a},
$$

where $H(A)$ is the entropy of categorical variable $A, a$ is a category of $A$, and $p_{a}$ is the proportion of observed values within that category (see Young \& Wasserman, 2001b, for a Web-based calculator that computes entropy). For our displays, we assume that categorization is based on the

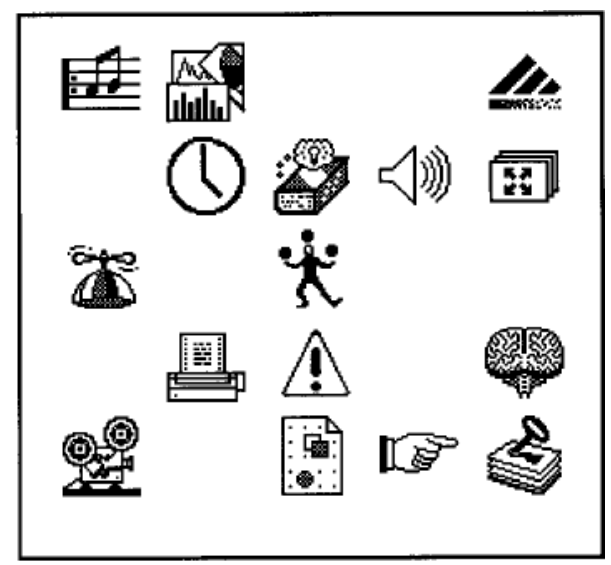

Figure 2. Same and different displays involving 16 icons distributed within a $5 \times 5$ array. 
obvious perceptual differences between the icons. When a display has 16 identical icons, entropy is 0 because there is no categorical variability; when a display has 16 different icons, entropy is 4 (the maximum for 16 items), indicating high categorical variability. If the pigeons' behavior is produced by the computation of entropy or a similar measure of categorical variability, changing the spatial orderliness of the icons in a display should have no effect on the pigeons' performance, because entropy (as computed by Equation 1) is entirely independent of orderliness.

How can we choose between these rival accounts? If same-different discrimination is based on a purely perceptual process, reducing or eliminating the spatial regularities in the same arrays should decrease the pigeon's discrimination accuracy; same arrays should now resemble different arrays.

One way to upset the spatial regularities of the items in an array is to disrupt the vertical and horizontal alignment of the computer icons (see Figure 3). According to a perceptual account, such disruption should impair discriminative performance because spatially disarranging the icons removes many of the regularities in the $4 \times 4$ same arrays (note that disarranging is a static manipulation, as is shown in Figure 3; no motion was used). Experiment 1 directly tested this prediction and yielded unequivocal results: Disarranging did not impair discriminative performance.

Experiment 2 disrupted the spatial regularities of the items in a display by mixing planar rotations of the icons. This manipulation was of particular interest because it not only provided a second look at the possible role of spatial orderliness in same-different discrimination, but also allowed us to determine whether pigeons perceived these displays as comprising 16 orientation-specific visual patterns or 16 orientation-invariant visual patterns. Our results were again unequivocal:Mixing planar rotations in an array did not impair discriminative performance.

These and other findings suggest that the pigeon's same-different discrimination is not measurably controlled by differences in the spatial orderliness of the same and the different arrays. Instead, the data suggest that the pigeon first discriminates the identities of the icons that constitute a display regardless of their precise position or their planar orientation en route to its determining the variability or entropy of the display.

\section{EXPERIMENT 1}

Pigeons that had previously been trained to discriminate between orderly $4 \times 4$ same and different arrays (see Figure 1) were later tested with disarranged $4 \times 4$ same and different arrays (see Figure 3 ). These disorderly testing arrays were created by disarranging each of the icons; each icon was displaced from its training location by a randomly determined distance, both vertically and horizontally. Disarranging the icons disrupted the spatial regularity in the original same displays; disarranging had a much smaller effect on the spatial regularity in the original different displays, because of the high degree of variability in icon size and shape in those displays.

\section{Method \\ Subjects \\ The subjects were 4 feral pigeons that were maintained at $85 \%$ of their free-feeding weights by controlled daily feeding. The pigeons had earlier been trained (Wasserman, Young, \& Nolan, 2000) to dis- criminate same from different arrays in which the icons were arranged in neat rows and columns (see Figure 1).}

\section{Apparatus}

The experiment used four specially constructed chambers that were identical to those in Young and Wasserman (1997). The chambers used touch-sensitive screens that were placed in front of a computer monitor to record the location of the pigeons' pecks. A brushed aluminum panel was placed directly in front of the touch screen to allow the pigeons access to circumscribed portions of a video monitor that was placed against one end of the chamber; pictorial stimuli were presented within the square center display area $(7 \times 7 \mathrm{~cm})$ and colored choice areas (buttons) were presented within the two round report regions (1.9- $\mathrm{cm}$ diameter) that were located $2.3 \mathrm{~cm}$ diagonally below each of the lower corners of the central opening. Food pellet reinforcers were dispensed into a small cup on the rear wall.
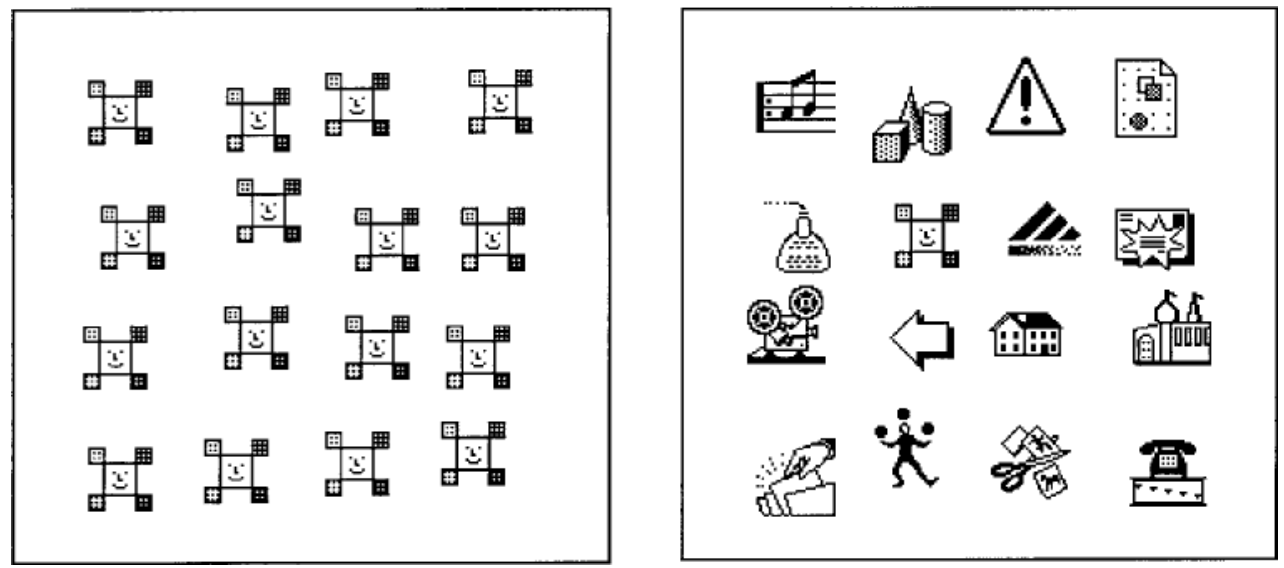

Figure 3. Examples of the spatially disarranged same and different arrays that were used in Experiment 1. 


\section{Visual Stimuli and Experimental Design}

Twenty-four highly distinguishable Macintosh icons were chosen as the total item pool. The icons were chosen from those in Young, Wasserman, and Dalrymple (1997). For any given same array, a single icon was randomly chosen, and it was used to make up an array of 16 identical icons. For the different arrays, 16 randomly chosen icons from the 24-icon pool were used, with no repetitions. The 16 same or 16 different icons were randomly distributed over the 16 locations in a $4 \times 4$ grid (this location randomization affected only the different arrays; see Figure 1 for representative same and different arrays).

In order to create disarranged versions of the displays, the 16 locations of the icons were each randomly displaced; the icon was moved between 1 and 6 pixels right or left and between 1 and 6 pixels up or down (6 pixels was approximately $0.2 \mathrm{~cm}$ ). The distance that an item was moved was restricted to 6 pixels or less in order to ensure that adjacent items would not touch if both were moved toward each other by the maximal amount.

\section{Procedure}

Training. Training sessions comprised 160 trials per daily session: 10 randomized blocks of 16 trials each, 8 with same arrays and 8 with different arrays.

Each trial began with illumination of the display area, with a white field containing a black cross in the center. A single peck anywhere in the display area turned on the icon array as a black-on-white picture. After a fixed number of pecks (determined separately for each bird) anywhere in the center display area, the icon array reversed to a white-on-black picture (to alert the bird to the availability of the report buttons), and the lower two report areas were lighted green (left) and red (right). Which response was correct for each array type was counterbalanced across subjects. A correct choice response blackened the display area and the report areas and delivered one or two 45-mg Noyes pigeon pellets (depending on each bird's $85 \%$ weight); an incorrect choice response blackened the report areas, turned off the houselight for 5 or $6 \mathrm{sec}$, and began a series of one or more correction trials that were not scored in data analyses. Intertrial intervals averaged $8 \mathrm{sec}$ (range, 6-10 sec).

Testing. Eight testing sessions were given. Testing sessions began with 32 warm-up trials with 16 same training arrays and 16 different training arrays. Thereafter, eight randomized blocks of 18 trials were given consisting of 8 same training arrays, 8 different training arrays, 1 disarranged same testing array, and 1 disarranged different testing array. Thus, the last 144 trials of the testing sessions consisted of 128 training trials with the orderly arrays of icons and 16 testing trials with disorderly arrays of icons.

Differential food reinforcement for left and right report responses was given on training trials (those trials involving the training arrays). Nondifferential reinforcement for left and right report responses was given on testing trials; food was given regardless of the pigeons' choice responses, so that repeated testing could be conducted without teaching the birds the correct responses to the testing arrays (if differential reinforcement had been given) or producing experimental extinction of responding to the testing arrays (if no reinforcement had been given). Thus, the designations correct and incorrect for choice responses on testing trials were for scoring purposes only. No correction trials were used on testing trials.

\section{Results}

Discrimination of same from different trials in the 5 days prior to testing averaged $86 \%, 91 \%, 88 \%$, and $87 \%$ correct for Birds $30 \mathrm{y}, 100 \mathrm{w}, 95 \mathrm{r}$, and $85 \mathrm{w}$, respectively. The testing performance of the 4 birds on the original and disarranged trials is shown in Figure 4. The birds responded correctly on a very high percentage of all of the trials, averaging $91 \%$; surprisingly, the pigeons were slightly more accurate on the disarranged trials than on the original trials.

For statistical analysis, the percentage correct scores were transformed using the logit function

$$
\frac{1}{2} \ln \frac{p}{100-p}
$$

The transformation was needed in order to ensure a nearly normal distribution; the transformation is highly recommended for percentage or proportion data (Cohen \& Cohen, 1983).

A full factorial, repeated measures analysis of variance (ANOVA) of the transformed percentage correct scores as a function of array organization (originalvs. disarranged)

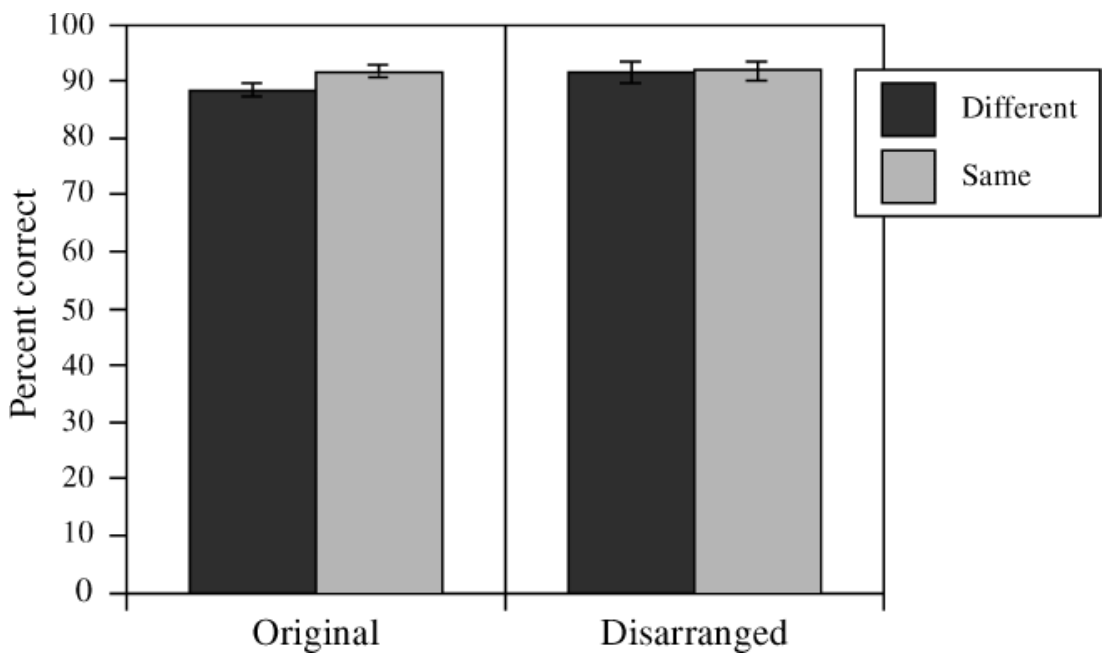

Figure 4. Discriminative performance to the original and the disarranged arrays of Experiment 1 . 


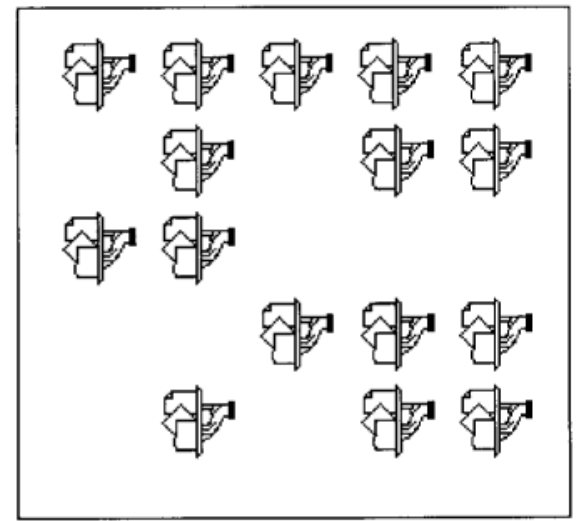

Same-Uniform $\left(270^{\circ}\right)$

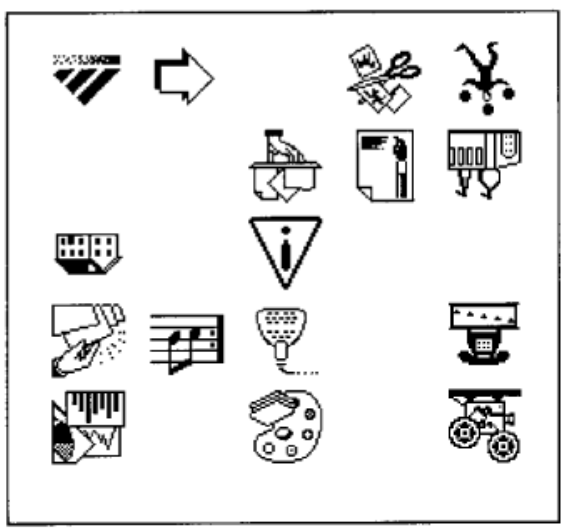

Different-Uniform $\left(180^{\circ}\right)$

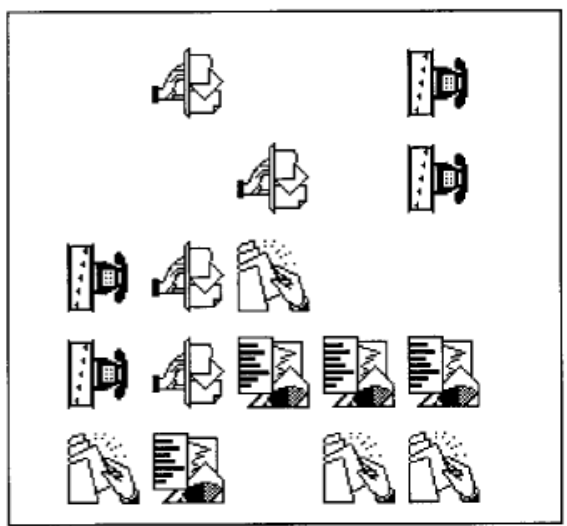

4-4-4-4-Uniform $\left(90^{\circ}\right)$

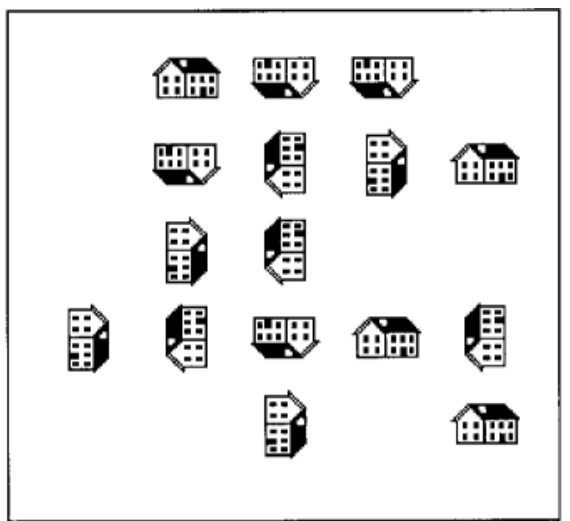

Same-Varied

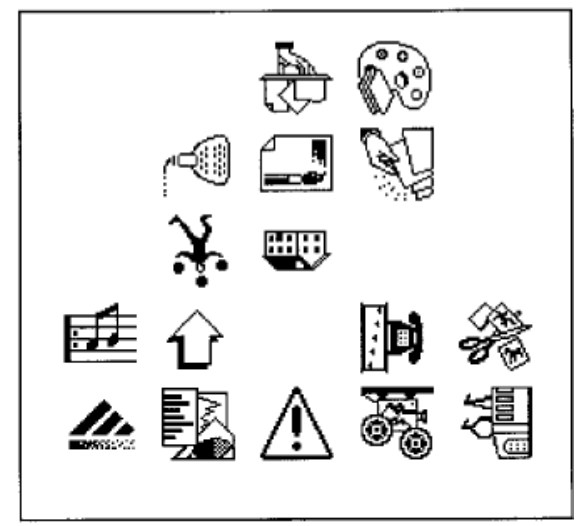

Different-Varied

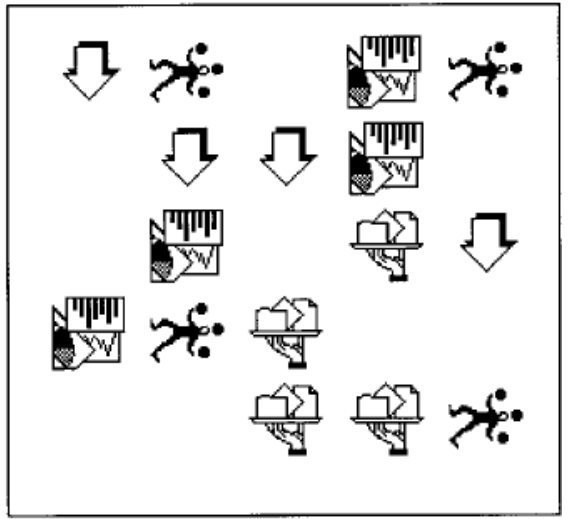

4-4-4-4-Varied

Figure 5. Examples of the many kinds of arrays that were used in Experiment 2.

and array type (same vs. different) revealed that none of the main effects or interactions approached statistical significance $\left(F_{\mathrm{S}}<1\right)$.

\section{Discussion}

In Experiment 1, we found that pigeons' discriminative responding was unaffected by disrupting the spatial regu- larities of the 16 visual items in a $4 \times 4$ array. By disarranging the icons within an array, our aim was to alter those spatial regularity differences that could have supported our pigeons' original discriminative performance. Evidently, the regular placement of the same icons in neat rows and columns was not necessary for our birds to distinguish the same arrays from the different arrays, thus sug- 
gesting that spatial regularity differences between these types of arrays may not have been the basis of our pigeons' original discriminative performance.

It is possible, however, that the absence of an effect may be due to the degree of disarranging that we used; perhaps more severe disarranging is necessary to disrupt the spatial orderliness of the arrays. Analyses of the spatial frequency profiles of the original and disarranged arrays revealed that disarranging produced large disruptions in the spatial regularities that were present in the same arrays (see http://www.siu.edu/departments/cola/psycho/faculty/ young/Research/SpatialFrequency.htm for a detailed discussion). Thus, our disarranging manipulation had a large impact on the spatial orderliness of the arrays, while having no measurable effect on the pigeons' discriminative performance.

\section{EXPERIMENT 2}

As a further exploration of both the generality of our pigeons' discriminative behavior and the plausibility of a spatial regularity account, in Experiment 2, we tested a new group of pigeons with arrays that contained a mixture of planar rotations of the icons at $0^{\circ}, 90^{\circ}, 180^{\circ}$, and $270^{\circ}$ after the birds had been trained to discriminate uniformly oriented same and different arrays (see the top two rows of Figure 5 for illustrations of these types of arrays). If the four different rotations of an icon are nevertheless judged to be identical, same trials should be similarly responded to whether the icons are uniformly or differently oriented; but if the four rotations of an icon are judged to be nonidentical, the pigeons should behave as if a same array with varied icon rotations is an array comprising four different types of icons (see the bottom row of Figure 5). Responding to the different arrays should be unaffected by the variation in icon rotations, because the different arrays already comprise 16 different kinds of icons.

The variation in icon rotations markedly disrupts the spatial regularity of a display; so a perceptual account predicts disruptions in discriminative performance that are specific to displays of identical icons. Varying the icon rotation of nonidentical icons should have no effect, because the icons are already perceived as being different from one another.

\section{Method}

\section{Subjects}

The subjects were 3 feral pigeons that were maintained at $85 \%$ of their free-feeding weights by controlled daily feeding. The pigeons had earlier been trained to discriminate same from different arrays in which 16 (of 24) icons were randomly located in 25 locations (a $5 \times 5$ array) for an unrelated study; the birds that were tested in Experiment 1 were no longer available for further training and testing.

\section{Apparatus}

The apparatus was identical to that used in Experiment 1.

\section{Procedure}

Visual stimuli. In order to ensure that we used only icons whose shapes markedly changed after planar rotation, highly symmetrical icons were eliminated from the item pool (e.g., the sun-like compass icon in Figure 1). Thus, the item pool was reduced from the original 24 on which the pigeons had been trained to 16 .

Arrays involving a uniformity of rotations (all of the icons within a display were presented at the same rotation) were used in a preliminary testing phase and in subsequent training (as represented by the same-uniform and different-uniform arrays of Figure 5). Arrays involving varied rotations and different distributions of icon types were used in testing. The testing arrays were same involving varied rotations (same-varied), different involving varied rotations (different-varied), a 4-4-4-4 distribution (four of one icon type, four of a second icon type, etc.) involving uniform rotations (4-4-4-uniform), and a 44-4-4 distribution involving varied rotations (4-4-4-4-varied). When the array comprised icons in various rotations, four of the icons were randomly chosen and presented at the $0^{\circ}$ rotation, four at $90^{\circ}$, four at $180^{\circ}$, and four at $270^{\circ}$. The only exception to the random choice rule was for the 4-4-4-4 arrays in which each of the four icon types was presented at the same rotation (e.g., brain $90^{\circ}$, brain $90^{\circ}$, brain $90^{\circ}$, brain $90^{\circ}$, house $270^{\circ}$, house $270^{\circ}$, house $270^{\circ}$, house $270^{\circ}$, etc.). Examples of the training and the testing arrays are shown in Figure 5.

The 4-4-4-4 arrays were included as additional baselines of comparison for the same-varied arrays. If the pigeons perceived the four rotations of the same icon (i.e., the icons that constitute the samevaried arrays) as involving four nonidentical pictures, their responding to the same-varied arrays should be equivalent to that observed to arrays involving a mixture of four nonidentical icons (i.e., a 4-4-4-4 array). If the pigeons perceived the different rotations to be of the same picture, their responding should be equivalent to that observed to a same-uniform array. Any responding between these extremes would suggest that rotating the icons made the icons appear less similar to one another than they were when they were presented at the same rotation, but more similar than if they were completely different icons.

Preliminary testing. The pigeons were originally trained to discriminate $5 \times 5$ same from $5 \times 5$ different arrays in which the icons were all presented at a nominal rotation (the $0^{\circ}$ rotation). Before training the pigeons with other uniform rotations (in order to eliminate their novelty for later testing), we first tested the birds for spontaneous transfer to displays in which all 16 icons were presented at the same rotation but this rotation was one of the untrained ones $\left(90^{\circ}, 180^{\circ}\right.$, or $\left.270^{\circ}\right)$.

Each session of testing involved 176 trials and began with 32 warmup trials containing 16 same- $0^{\circ}$ (a same-uniform display in which all of the icons were presented at a $0^{\circ}$ rotation) and 16 different $-0^{\circ}$ displays. The remaining 144 trials consisted of four blocks of 36 trials: 15 same $-0^{\circ}, 15$ different $-0^{\circ}$, and 1 each of same $-90^{\circ}$, same $-180^{\circ}$, same- $270^{\circ}$, different $-90^{\circ}$, different- $180^{\circ}$, and different $-270^{\circ}$. Differential food reinforcement was given on the $0^{\circ}$ training trials; nondifferential reinforcement was given on the $90^{\circ}, 180^{\circ}$, or $270^{\circ}$ testing trials. The birds received eight testing sessions.

Training. After preliminary testing, each pigeon was trained with same-uniform and different-uniform arrays under differential reinforcement (the pigeons received reinforcement only for a correct response). In each display, all 16 icons were presented at one of four rotations $\left(0^{\circ}, 90^{\circ}, 180^{\circ}\right.$, or $\left.270^{\circ}\right)$; all of the icons within a display were presented at the same rotation. This procedure was used in order to ensure that the new rotations would not be novel during subsequent testing. A session comprised 10 blocks of 16 trials; a block involved two presentations each of same $-0^{\circ}$, same $-90^{\circ}$, same $-180^{\circ}$, same $-270^{\circ}$, different $-0^{\circ}$, different $-90^{\circ}$, different- $180^{\circ}$, and different- $270^{\circ}$. Once a pigeon's accuracy was at least $85 \%$ and similar for the four rotations (as judged by individual ANOVAs conducted each day with a criterion of $p>.10$ for both the main effect of rotation and its interaction with stimulus type [same vs. different]), it was moved to testing.

Testing. The birds received eight testing sessions. Testing sessions began with 32 warm-up trials with 16 same-uniform arrays 
and 16 different-uniform arrays; responding to these arrays was not scored. Thereafter, four randomized blocks of 36 trials were given, consisting of four presentations each of the same- $0^{\circ}$, same $-90^{\circ}$, same $-180^{\circ}$, same $-270^{\circ}$, different- $0^{\circ}$, different- $90^{\circ}$, different- $180^{\circ}$, and different $-270^{\circ}$ training arrays, and one presentation each of the same-varied, different-varied, 4-4-4-4-uniform, and 4-4-4-4varied arrays. Thus, the last 144 trials of the testing sessions consisted of 128 trials with training arrays and 16 trials with testing arrays. Differential food reinforcement for left and right report responses was given on the training trials; nondifferential reinforcement for left and right report responses was given on the testing trials.

\section{Results and Discussion}

Preliminary testing revealed strong but incomplete generalization to the uniform displays involving novel rotations. Discrimination averaged $93 \%$ for the $0^{\circ}$ rotations and $79 \%$ for the novel rotations (with no significant differences among the three rotations). A repeated measures ANOVA of logit percentage correct as a function of rotation novelty (trained vs. untrained) revealed a significant main effect of novelty $\left[F(1,2)=111.85, M S_{\mathrm{e}}=0.6325, p<.01\right]$. Individual binomial tests revealed that accuracy to the $0^{\circ}$ rotations and the novel rotations exceeded the chance score of $50 \%$ correct (one-tailed $p s<.01$ ). The pigeons thus readily transferred their prior training to displays involving novel rotations of the trained icons, but the lower accuracy indicated that the novelty of the rotations was noticed.

Discrimination training with the novel rotations took only 5, 7, and 6 days for Birds 91w, 32y, and 75y, respectively; discrimination of same from different arrays during the 5 days before testing averaged $93 \%, 89 \%$, and $86 \%$ correct. The pigeons thus quickly improved their discriminative behavior to displays comprising familiar icons presented at novel rotations.
Varying the rotation of the icons within a display had a very small effect on the pigeons' performance (see Figure 6). Most important, the pigeons judged planar rotations of the same icon to be identical to one another; the same-varied arrays were judged to be much more similar to the sameuniform arrays than they were to either the 4-4-4-4varied or the 4-4-4-4-uniform arrays. Varying the rotation of the icons also had no effect on whether different icons were perceived as different from one another; these stimuli were judged to be different from one another regardless of the rotations of the individual icons. The 4-44-4 displays of either varied or uniform icon rotations yielded a percentage of different responses that fell between that produced by the same and that produced by the different arrays. This percentage of different report responses is anticipated by the intermediate degree of variability or entropy of these displays (Young \& Wasserman, 1997).

In order to confirm the effects of rotation on discriminative performance, we conducted a full factorial, repeated measures ANOVA on the logit percentage of different responses as a function of array type (same, different, and 4-4-4-4) and rotation composition (uniform vs. varied). Responding differed as a function of the array type $\left[F(2,4)=125.14, M S_{\mathrm{e}}=3.108, p<.0001\right]$, but neither the main effect of rotation composition nor the array type $X$ rotation composition interaction was statistically significant $\left(F_{\mathrm{S}}<1\right)$. Responding to the 4-4-4-4 arrays was statistically different from that to both the same and the different arrays ( $p<.05$, Newman-Keuls).

Experiment 2 thus revealed a high degree of stimulus generalization across item rotation. Varying the rotation of the icons within either same or different arrays had no discernible impact on the pigeons' discriminative perfor-

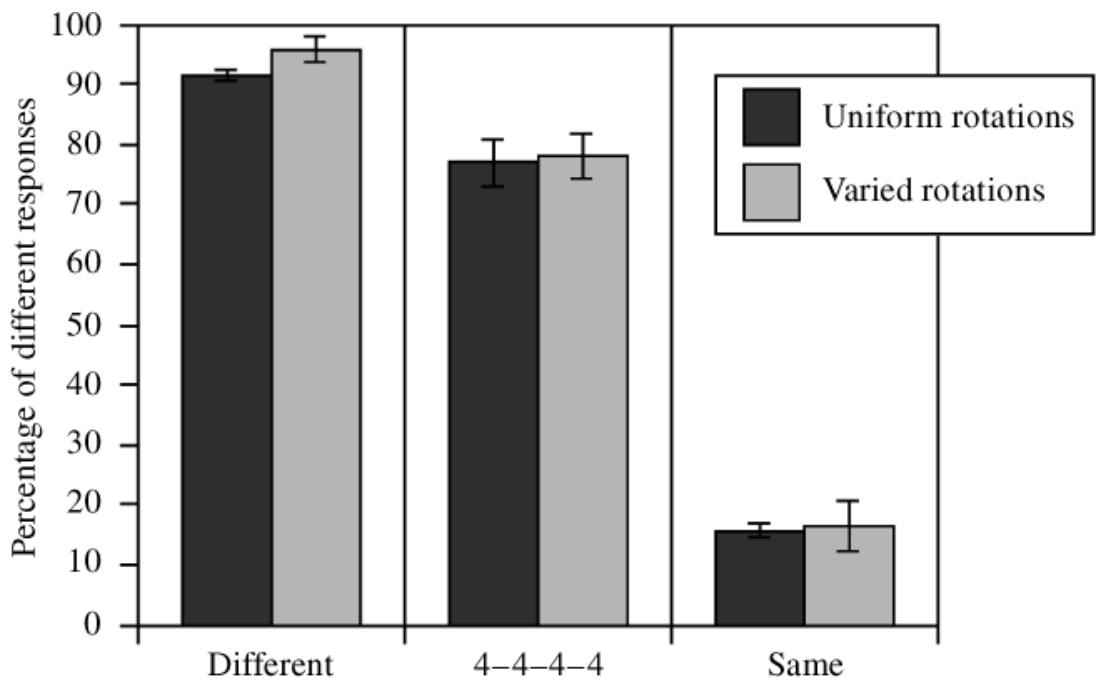

Figure 6. Discriminative performance for the same, different, and 4-4-4-4 arrays of Experiment 2 with both consistent (uniform) and inconsistent (varied) within-display rotations of the icons. 
mance despite the fact that such rotation decidedly upset icon regularity, especially on same arrays.

\section{GENERAL DISCUSSION}

The present pair of experiments evaluated the pigeons' possible use of spatial regularity or orderliness in solving a same-different discrimination. Our results clearly showed that disrupting the spatial regularity of displays of 16 visual items had no measurable effect on the pigeons' discriminative performance. Neither spatially disarranging (Experiment 1) nor varying the planar rotation of those items (Experiment 2) adversely affected the pigeons' choice responding.

As well, our results showed that arrays of same visual items were treated equivalently whether those arrays contained uniformly oriented icons or varied planar rotations of the icon at $0^{\circ}, 90^{\circ}, 180^{\circ}$, and $270^{\circ}$ (Experiment 2). So, pigeons not only generalize their same-different discrimination performance to novel visual items (Wasserman et al., 1995; Young \& Wasserman, 1997), but also deem the rotation of the items to be irrelevant to judging the sameness or differentness of the items in a display (Experiment 2).

Our appeal to the processing of visual display variability or entropy represents a clear and concise account of the pigeon's same-different discrimination across a wide range of experimental investigations (Wasserman et al., 2000; Young \& Wasserman, 1997; Young, Wasserman, \& Garner, 1997). It has also found support in studies of same-different discrimination in baboons (Wasserman, Fagot, \& Young, 2001) and human beings (Young \& Wasserman, 2001a). The present experiments and several others by Wasserman et al. (1995), Young and Wasserman (1997), and Young,
Wasserman, and Dalrymple (1997) thus provide considerable evidence that same-different discriminative behavior is conceptual in nature and is not simply the product of a low-level perceptual process.

\section{REFERENCES}

Cohen, J., \& Cohen, P. (1983). Applied multiple regression/correlation analysis for the behavioral sciences (2nd ed.). Hillsdale, NJ: Erlbaum.

Shannon, C. E., \& WeAVER, W. (1949). The mathematical theory of communication. Urbana: University of Illinois Press.

Wasserman, E. A., Fagot, J., \& Young, M. E. (2001). Same-different conceptualization by baboons (Papio papio). Journal of Comparative Psychology, 115, 42-52.

Wasserman, E. A., Hugart, J. A., \& Kirkpatrick-Steger, K. (1995). Pigeons show same-different conceptualization after training with complex visual stimuli. Journal of Experimental Psychology: Animal Behavior Processes, 21, 248-252.

Wasserman, E. A., Young, M. E., \& Nolan, B. C. (2000). Display variability and spatial organization as contributors to the pigeon's discrimination of complex visual stimuli. Journal of Experimental Psychology: Animal Behavior Processes, 26, 133-143.

Young, M. E., \& Wasserman, E. A. (1997). Entropy detection by pigeons: Response to mixed visual displays after same-different discrimination training. Journal of Experimental Psychology: Animal Behavior Processes, 23, 157-170.

Young, M. E., \& WAsserman, E. A. (2001a). Entropy and variability discrimination. Journal of Experimental Psychology: Learning, Memory, \& Cognition, 27, 278-293.

Young, M. E., \& Wasserman, E. A. (2001b). Stimulus control in complex arrays. In R. Cook (Ed.), Avian visual cognition [On-line]. Available: http://www.pigeon.psy.tufts.edu/avc.

Young, M. E, Wasserman, E. A., \& Dalry mple, R. M. (1997). Memorybased same-different conceptualization by pigeons. Psychonomic Bulletin \& Review, 4, 552-558.

Young, M. E., Wasserman, E. A., \& Garner, K. L. (1997). Effects of number of items on the pigeon's discrimination of same from different visual displays. Journal of Experimental Psychology: Animal Behavior Processes, 23, 491-501. 